
\title{
25 Research Square \\ The role of routine laboratory tests after unilateral total knee arthroplasty
}

\section{An an $\mathrm{Li}$}

First Affiliated Hospital of Nanchang University

\section{Yu Zhang}

First Affiliated Hospital of Nanchang University

\section{Hao zhang}

fouth affiliated hospital of nanchang university

\section{Shi-ning xiao}

First Affiliated Hospital of Nanchang University

xin-hua long

Affiliated Hospital of Nanchang University

\section{Shi-jiang Wang}

Affiliated Hospital of Nanchang University

yang zhou ( $\sim$ zoya1323@163.com)

Affiliated Hospital of Nanchang University

\section{Research Article}

Keywords: Unilateral total knee arthroplasty, risk factor, Postoperative laboratory tests, clinical intervention

Posted Date: February 28th, 2022

DOI: https://doi.org/10.21203/rs.3.rs-1340814/v1

License: (c) (1) This work is licensed under a Creative Commons Attribution 4.0 International License. Read Full License 


\section{Abstract}

\section{Background}

Recent studies suggest that routine laboratory tests are not required within 1 day after partial knee arthroplasty. This study aimed to evaluate the utility of routine postoperative laboratory tests after initial unilateral total knee arthroplasty(TKA) in an Asian population and to identify risk factors associated with abnormal test results.

\section{Methods}

The clinical data of patients who underwent original unilateral TKA from 2015 to 2020 were retrospectively analyzed. Patient characteristics and laboratory test values were recorded. Multivariate binary logistic regression analysis was also performed to identify risk factors associated with 3 abnormal laboratory values.

\section{Results}

A total of 713 patients were included in this study and underwent relevant laboratory tests within 3 days of TKA surgery. However, only a small proportion of patients with anemia (8.1\%), hypoalbuminemia (9.9 $\%)$, and abnormal serum potassium levels (3.4\%) required clinical intervention after surgery. Binary logistic regression analysis revealed that independent risk factors for postoperative blood transfusion in TKA patients included preoperative hemoglobin level, estimated blood loss, and age. Related risk factors for postoperative albumin supplementation were preoperative albumin level, intraoperative blood loss and operation time. In addition, lower body mass index (BMI) and preoperative hypokalemia were found to be potential risk factors for postoperative potassium supplementation.

\section{Conclusion}

Since more than $90 \%$ of abnormal postoperative laboratory tests do not require clinical intervention, the authors believe that it is of little significance for patients with primary unilateral TKA to receive routine laboratory tests after surgery. However, in patients with established risk factors, postoperative laboratory testing is still necessary.

\section{Introduction}

One of the most common and successful surgeries today, total knee arthroplasty (TKA) is one of the leading options for the treatment of end-stage knee osteoarthritis ${ }^{1}$, allowing surgeons to restore a patient's knee joint function and reduce pain ${ }^{2}$. With increasing aging, more and more patients will receive TKA.

Laboratory tests are an essential diagnostic tool in traditional clinical decision-making, and blood testing of patients after surgery is well established in routine clinical practice, especially after major orthopaedic 
surgery ${ }^{3}$, such as TKA. Postoperative laboratory tests are performed to prevent the omission of critical clinical details and potentially serious complications ${ }^{4}$. In the past decade, the advent of the concept of fast recovery and the significant improvement of perioperative care pathways have greatly shortened the length of hospital stay and the incidence of postoperative complications ${ }^{5,6}$. At the same time, the widespread use of tranexamic acid also greatly reduces the risk of blood loss and the chance of blood transfusion ${ }^{7,8}$. Therefore, many scholars began to ask whether it is necessary to perform routine laboratory tests in uncomplicated patients. Several studies have confirmed that there is no need for routine laboratory blood tests in patients without risk factors after surgery such as total hip replacement ${ }^{4,9}$, shoulder replacement ${ }^{10}$, and partial knee arthroplasty ${ }^{11}$. However, few studies have reported the need for routine laboratory testing in patients after TKA surgery.

The medical status of patient groups in different regions of the world varies widely, and malnutrition problems such as anemia and hypoalbuminemia remain high in Asian elderly, so reassessment of the utility of routine blood tests after initial postoperative Unilateral THA is necessary in Asian populations. Therefore, this study aimed to assess the necessity of routine postoperative laboratory tests after initial elective unilateral TKA, identify risk factors associated with abnormal laboratory tests, and provide better guidance for clinical work.

\section{Methods}

\section{Study Design}

All subjects included in the study were from patients who underwent TKA surgery in our hospital between 2015 and 2020. Patients undergoing bilateral total knee arthroplasty were excluded. In addition, patients with infections, malignant tumors, and rheumatoid osteoarthritis were also excluded from the study.

\section{Date collection}

The data collected included demographic and clinical characteristics of all patients (sex, age, body mass index, and preoperative comorbidities), surgical factors (intraoperative blood loss, operative time), preoperative and postoperative laboratory parameters (liver function, renal function, electrolytes, and inflammatory markers) and the incidence of abnormal postoperative laboratory markers and related interventions (blood transfusion, albumin supplementation, and potassium supplementation). At the same time, the risk factors of abnormal postoperative laboratory indicators requiring intervention are analyzed.

The reference ranges of all laboratory test indicators in this study are listed in table1. The basic requirements for clinical intervention for abnormal laboratory indicators after surgery ${ }^{12}$ : hemoglobin < $70 \mathrm{~g} / \mathrm{L}$ or symptom-

atic anemia with a hemoglobin level of $<100 \mathrm{~g} / \mathrm{L}$, albumin $<30 \mathrm{~g} / \mathrm{L}$, serum potassium $<3.5 \mathrm{mmol} / \mathrm{L}$. 


\section{Surgical technique}

The surgical incision starts $6-10 \mathrm{~cm}$ above the patella and goes down $1-2 \mathrm{~cm}$ to the tibial tubercle. The medial $1 / 3$ of the insertion point of the patellar ligament was stripped, the patella was reversed laterally, the anterior and posterior cruciate ligaments were cut off, the meniscus, the hyperplastic synovium and the marginal osteophyte were removed. Intramedullary or extramedullary positioning by installing an osteotomy guide, then osteotomy of the femur and tibia, inserting the appropriate size joint prosthesis, and moving the knee joint to check the range of motion. 3 days after the operation, patients can move around with crutches.

\section{Statistical analysis}

Qualitative variables were analyzed by chi-square test, and quantitative variables were analyzed by independent sample t-test. Use binary logistic regression analysis to find risk factors for abnormal postoperative experimental indicators that require clinical intervention, and then evaluate the predictive value of risk factors and find cut-off values by drawing ROC curves. All data were analyzed using SPSS version 26 (Inc., Chicago, IL). A p value of less than 0.05 was considered statistically significant.

\section{Result}

\section{Laboratory test characteristics}

Abnormal laboratory parameters were collected in 713 patients after unilateral TKA. The main abnormal indicators of all laboratory tests after surgery were serum albumin, accounting for $96.5 \%$, followed by hemoglobin(95.8\%) and creatinine (45.2\%) concentration. However, only a small proportion of postoperative patients with abnormal laboratory parameters received appropriate interventions, including $8.1 \%$ of patients with low hemoglobin, $9.9 \%$ of patients with low albumin and $3.4 \%$ of patients with hypokalemia(Table 2).

\section{Risk factors for patients requiring postoperative clinical treatment}

\section{Risk factors for patients requiring postoperative blood transfusion}

Univariate analysis of the patient data between the two groups (with and without blood transfusion) showed no significant differences in gender, preoperative comorbidities, and body mass index (BMI). However, the age $(p=0.001)$, operative time $(p=0.013)$ and intraoperative blood loss $(p<0.001)$ in the postoperative transfusion group were greater than those in the non-transfusion group, and the preoperative hemoglobin $(p<0.001)$ was significantly lower than that in the non-transfusion group(Table $3)$. Binary logistic regression analysis showed that age $(O R=1.148, P<0.001)$, intraoperative blood loss $(O R=1.008, P<0.001)$ and preoperative hemoglobin $(O R=0.665, P<0.001)$ were independent risk factors for postoperative blood transfusion in TKA patients(Table 4).

Risk factors for patients requiring postoperative albumin supplementation 
Univariate analysis showed that there were significant differences between the supplemented albumin group and the non-supplemented group in gender, smoking, drinking, preoperative albumin, intraoperative blood loss, and operative time(Table 5). Further binary logistic regression analysis found that preoperative albumin(OR=0.700, $\mathrm{P}<0.001)$, operation time( $\mathrm{OR}=1.011, \mathrm{P}<0.001)$ and intraoperative blood loss $(\mathrm{OR}=1.004, \mathrm{P}=0.037)$ were independent risk factors for postoperative albumin supplementation in TKA patients(Table 6).

\section{Risk factors for patients requiring postoperative potassium supplementation}

Univariate and binary logistic regression analysis found that $\mathrm{BMI}(\mathrm{OR}=1.191, \mathrm{P}=0.007)$ and preoperative serum potassium( $(\mathrm{R}=0.019, \mathrm{P}<0.001)$ were independent risk factors for postoperative serum potassium supplementation in TKA patients(Table 7 and Table 8).

\section{Diagnostic accuracy of risk factors for predicting postoperative clinical treatment}

Assessing the diagnostic value of risk factors for clinical intervention after TKA by drawing receiver operating characteristic(ROC) curves and determining cut-off values for risk factors. The larger the area under the curve(AUC), the higher the prediction accuracy of its risk factors. The results are presented in Table 9. The results showed that the preoperative hemoglobin level had the highest accuracy in predicting blood transfusion (Auc=0.933, $\mathrm{P}<0.001$ ).

\section{Discussion}

In recent years, due to the frequent occurrence of medical accidents, many medical workers have made many patients perform excessive routine laboratory tests out of their own protection. This not only causes a great financial burden to patients, but also an unreasonable control of medical resources. Many studies $^{4,13}$ have shown that routine postoperative laboratory tests are not needed because they do not serve a certain degree of clinical value. However, it is necessary for those patients with risk factors to undergo postoperative laboratory testing ${ }^{4,14}$. Many studies suggest that inappropriate laboratory testing should be reduced to better improve the quality of care and reduce healthcare costs. The purpose of this study was to define when routine laboratory testing in patients following knee arthroplasty may or may not be necessary.

Laboratory tests are very helpful in the diagnosis and progression of the disease, but not the more the better. In his research, Gerald et al. ${ }^{15}$ revealed that the more laboratory tests a patient is subjected to, the more likely it is that an abnormality will eventually be found. To a certain extent, however, if the laboratory test results do not have clinical reference value, then the test is not necessary. Many studies have shown that routine postoperative laboratory tests are not required in most cases unless risk factors are present $3,4,13,14,16,17$. Li et al. ${ }^{14}$ showed that nearly $50 \%$ of patients undergoing high tibial osteotomy had abnormal postoperative laboratory results, but less than $4 \%$ required clinical intervention, suggesting that routine postoperative serology is not required for the majority of patients laboratory test. In our study, up 
to $95 \%$ of postoperative test results were abnormal, but only about $8 \%$ required intervention, indicating that in most cases, patients with TKA do not need routine laboratory tests after surgery.

Dai et $\mathrm{al}^{18}$ reported that age and low hemoglobin on admission were important risk factors for perioperative blood transfusion. Armin et al ${ }^{19}$ retrospectively analyzed 8461 elderly patients over 65 years of age who underwent hip replacement in the United States in 2016 and found that older age and preoperative anemia were independent risk factors for postoperative blood transfusion. Cao et al ${ }^{20}$ retrospectively analyzed 414 patients with total hip arthroplasty and 1147 patients with total knee arthroplasty and found that increased intraoperative blood loss was a risk factor for postoperative blood transfusion. Consistent with previous studies, in our study, older age, low preoperative hemoglobin, and increased intraoperative blood loss were found to be significant risk factors for postoperative blood transfusions.

In a retrospective study, Wu et $\mathrm{al}^{13}$. analyzed 213 patients undergoing dance hip arthroplasty and showed that the incidence of postoperative albumin was $72.3 \%$, of which $19.7 \%$ received clinical intervention. Our study found that the incidence of postoperative abnormal serum albumin was as high as $96.5 \%$, but only $9.9 \%$ of patients received clinical intervention, which was lower than reported by Wu et al. In another study, Wu et al ${ }^{12}$. found that long operative time and low preoperative albumin levels were risk factors for postoperative albumin supplementation, which is consistent with our findings. In addition, we also found that increased intraoperative blood loss is also an important factor for albumin supplementation, as increased operative time increases intraoperative blood loss in patients.

$\mathrm{Li}$ et $\mathrm{al}^{14}$. analyzed 482 patients undergoing high tibial osteotomy and found that the proportion of postoperative electrolyte abnormalities was low, including 3.5\% of abnormal serum potassium. Preoperative serum potassium concentration below $3.45 \mathrm{mmol} / \mathrm{L}$ was an independent risk factor for postoperative potassium supplementation. In a retrospective study, Jordan et al ${ }^{10}$ analyzed data from 213 patients undergoing shoulder arthroplasty and found that postoperative electrolyte abnormalities were strongly associated with lower BMI. Consistent with the above findings, in this study, we found that postoperative patients had a lower probability of electrolyte abnormalities, preoperative serum potassium concentration less than $3.68 \mathrm{mmol} / \mathrm{I}$, and BMI less than 21.89 were important risk factors for postoperative potassium supplementation.

There are some shortcomings in this study. First of all, this is a single-center retrospective study, there are some missing data, and the number of included studies is limited, which will lead to biased results to a certain extent. Second, there are certain differences among different surgeons in intervening on abnormal postoperative laboratory tests, which will also bias the final analysis results. Therefore, it is necessary to conduct a multicenter study with a larger sample to verify the effectiveness of these risk factors in predicting the need for clinical intervention in patients with abnormal laboratory tests after TKA.

\section{Conclusion}


In patients undergoing primary TKA, most laboratory findings tend to be abnormal; however, most abnormalities are borderline and very few patients require further clinical intervention. Therefore, we concluded that routine scheduling of postoperative laboratory tests after TKA surgery is not necessary. However, in patients with established risk factors, routine postoperative laboratory tests are still necessary. For patients $>69.5$ years old, intraoperative blood loss $>225 \mathrm{ml}$, and preoperative hemoglobin < $111.5 \mathrm{~g} / \mathrm{l}$, we recommend elective complete blood count after operation. For patients with preoperative albumin $<42.85 \mathrm{~g} / \mathrm{l}$ and operation time $>152.5$ minutes, intraoperative albumin infusion can be considered. For patients with $\mathrm{BMI}<21.89 \mathrm{~kg} / \mathrm{m}^{2}$ and preoperative serum potassium $<3.68 \mathrm{~g} / \mathrm{l}$, basal metabolic group should be considered after operation. Our inferences above need to be further validated with larger sample studies.

\section{Declarations}

\section{Ethics approval and consent to participate}

This retrospective study passed the ethics review of the Medical Research Ethics Committee of the First Affiliated Hospital of Nanchang University. The experimental protocol was approved by First Affiliated Hospital of Nanchang University. All research methods were carried out in accordance with the Declaration of Helsinki. And All clinical data acquisitions were obtained with the informed consent of the patients.

\section{Consent for publication}

Not applicable.

\section{Availability of data and materials}

The datasets generated during the current study are not publicly available due to the article has not been published, but are available from the corresponding author(Yang zhou, E-mail: zoya1323@163.com)on reasonable request.

\section{Competing interests}

No conflict of interest

\section{Funding}

This work was supported by the Key Research and Development Program of Jiangxi Province, China(No.20202BBGL73015,20203BBG73045)

\section{Authors' contributions}

An-an li wrote the main manuscript text. Yu zhang analyzed the data. Hao fan collected data.Shi-ning xiao, Xin-hua long and Shi-jiang wang prepared tables1-9. Yang zhou proposed research ideas and 
reviewed articles.

\section{Acknowledgements}

Not applicable

\section{Authors' information}

An-an li: Department of Orthopedics of the First Affiliated Hospital of Nanchang University; Jiangxi, P.R. China.

E-mail: 2460627147@qq.com.

Yu zhang: Department of Orthopedics of the First Affiliated Hospital of Nanchang University; Jiangxi, P.R. China.

E-mail: jinxiangyu1989@163.com.

Hao zhang: Fourth Clinical Medical College of Nanchang University, Jiangxi, P.R. China.

E-mail: ncusuqyzxzh@163.com.

Shi-ning xiao: Department of Orthopedics of the First Affiliated Hospital of Nanchang University; Jiangxi, P.R. China.

E-mail: 1078047637@qq.com.

Xin-hua long: Department of Emergency of the First Affiliated Hospital of Nanchang University; Jiangxi, P.R. China.

E-mail: longxin1234@126.com.

Shi-jiang wang: Department of Orthopedics of the First Affiliated Hospital of Nanchang University; Jiangxi, P.R. China.

E-mail:673614985@qq.com.

Yang zhou: Department of Orthopedics of the First Affiliated Hospital of Nanchang University; Jiangxi, P.R. China.

E-mail: zoya1323@163.com.

\section{References}

1. Thacoor A, Sandiford N. Cryotherapy following total knee arthroplasty: What is the evidence? J Orthop Surg (Hong Kong). 2019;27(1):230949901983275. doi:10.1177/2309499019832752 
2. Papas PV, Congiusta D, Cushner FD. Cementless versus Cemented Fixation in Total Knee Arthroplasty. J Knee Surg. 2019;32(07):596-599. doi:10.1055/s-0039-1678687

3. Wu XD, Xiao PC, Zhu ZL, Liu JC, Li YJ, Huang W. The necessity of routine postoperative laboratory tests in enhanced recovery after surgery for primary hip and knee arthroplasty: A retrospective cohort study protocol. Medicine. 2019;98(18):e15513. doi:10.1097/MD.0000000000015513

4. Wu XD, Zhu ZL, Xiao PC, Liu JC, Wang JW, Huang W. Are Routine Postoperative Laboratory Tests Necessary After Primary Total Hip Arthroplasty? The Journal of Arthroplasty. 2020;35(10):28922898. doi:10.1016/j.arth.2020.04.097

5. Luzzi AJ, Fleischman AN, Matthews CN, Crizer MP, Wilsman J, Parvizi J. The "Bundle Busters": Incidence and Costs of Postacute Complications Following Total Joint Arthroplasty. The Journal of Arthroplasty. 2018;33(9):2734-2739. doi:10.1016/j.arth.2018.05.015

6. SooHoo NF, Farng E, Lieberman JR, Chambers L, Zingmond DS. Factors That Predict Short-term Complication Rates After Total Hip Arthroplasty. Clinical Orthopaedics \& Related Research. 2010;468(9):2363-2371. doi:10.1007/s11999-010-1354-0

7. Whiting DR, Duncan CM, Sierra RJ, Smith HM. Tranexamic Acid Benefits Total Joint Arthroplasty Patients Regardless of Preoperative Hemoglobin Value. The Journal of Arthroplasty. 2015;30(12):2098-2101. doi:10.1016/j.arth.2015.05.050

8. Evangelista PJ, Aversano MW, Koli E, et al. Effect of Tranexamic Acid on Transfusion Rates Following Total Joint Arthroplasty. Orthopedic Clinics of North America. 2017;48(2):109-115. doi:10.1016/j.ocl.2016.12.001

9. Halawi MJ, Plourde JM, Cote MP. Routine Postoperative Laboratory Tests Are Not Necessary After Primary Total Hip Arthroplasty. The Journal of Arthroplasty. 2019;34(3):538-541. doi:10.1016/j.arth.2018.11.037

10. Paynter JW, Raley JA, Kyrkos JG, et al. Routine postoperative laboratory tests are unnecessary after primary reverse shoulder arthroplasty. Journal of Shoulder and Elbow Surgery. 2020;29(8):16561664. doi:10.1016/j.jse.2019.12.023

11. Shaner JL, Karim AR, Casper DS, Ball CJ, Padegimas EM, Lonner JH. Routine Postoperative Laboratory Tests Are Unnecessary After Partial Knee Arthroplasty. The Journal of Arthroplasty. 2016;31(12):2764-2767. doi:10.1016/j.arth.2016.05.052

12. Wu XD, Zhu ZL, Xiao PC, Liu JC, Wang JW, Huang W. Are Routine Postoperative Laboratory Tests Necessary After Primary Total Hip Arthroplasty? The Journal of Arthroplasty. 2020;35(10):28922898. doi:10.1016/j.arth.2020.04.097

13. Wu XD, Liu JC, Li YJ, Wang JW, Qiu GX, Huang W. The necessity of routine postoperative laboratory tests after total hip arthroplasty for hip fracture in a semi-urgent clinical setting. J Orthop Traumatol. 2020;21(1):19. doi:10.1186/s10195-020-00559-3

14. Li H bo, Nie S, Lan M, Liao X gen, Tang Z ming. Re-evaluating the necessity of routine laboratory tests after high tibial osteotomy surgery. BMC Musculoskelet Disord. 2021;22(1):720. doi:10.1186/s12891-021-04608-8 
15. Smetana GW, Macpherson DS. The case against routine preoperative laboratory testing. Medical Clinics of North America. 2003;87(1):7-40. doi:10.1016/S0025-7125(02)00147-5

16. Lin JM, Cao ZY, Peng AF, et al. Are Routine Postoperative Laboratory Tests Really Necessary After Lumbar Spinal Surgery? World Neurosurgery. 2019;124:e748-e754. doi:10.1016/j.wneu.2018.12.214

17. Zhuang TF, Huan SW, Luo SM, et al. The role of routine postoperative laboratory tests following hip hemiarthroplasty for an elderly femoral neck fracture. BMC Musculoskelet Disord. 2021;22(1):806. doi:10.1186/s12891-021-04698-4

18. Dai CQ, Wang $L H$, Zhu YQ, et al. Risk factors of perioperative blood transfusion in elderly patients with femoral intertrochanteric fracture. Medicine. 2020;99(15):e19726. doi:10.1097/MD.0000000000019726

19. Arshi A, Lai WC, Iglesias BC, et al. Blood transfusion rates and predictors following geriatric hip fracture surgery. HIP International. 2021;31(2):272-279. doi:10.1177/1120700019897878

20. Bedrosian I, Faries MB, Iv DG, et al. Incidence of Sentinel Node Metastasis in Patients With Thin Primary Melanoma ( $₫ 1 \mathrm{~mm}$ ) With Vertical Growth Phase. Ann Surg Oncol. 2000;7(4):6.

\section{Tables}

Table 1. The normal reference ranges for laboratory values 


\begin{tabular}{|ll|}
\hline Laboratory Tests & Reference range \\
\hline Routine blood test & \\
\hline Hemoglobin (g/L) & $130-175$ \\
\hline Platelets (*10^9/L) & $125-350$ \\
\hline Liver function test & \\
\hline Alanine aminotransferase (IU/L) & $9-50$ \\
\hline Aspartate aminotransferase (IU/L) & $15-40$ \\
\hline Albumin (g/L) & $40-55$ \\
\hline Renal function test & \\
\hline Creatinine (umol/L) & $57-97$ \\
\hline Blood urea nitrogen (mmol/L) & $3.1-8.0$ \\
\hline Inflammation indicators test & \\
\hline Erythrocyte sedimentation rate(mm/h) & $0-20$ \\
\hline C-reactive protein(mg/l) & $0-8$ \\
\hline Electrolytes & \\
\hline Serum sodium (mmol/L) & $137-147$ \\
\hline Serum potassium serum potassium (mmol/L) & $3.5-5.3$ \\
\hline Serum calcium (mmol/L) & $2.11-2.52$ \\
\hline
\end{tabular}

Table 2. Results of Routine Laboratory Tests for Patients Undergoing TKA Surgery 


\begin{tabular}{|c|c|c|}
\hline $\begin{array}{l}\text { Laboratory Test } \\
(n=713)\end{array}$ & $\begin{array}{l}\text { Abnormal Laboratory Test } \\
\text { Result (n; \%) }\end{array}$ & $\begin{array}{l}\text { Postoperative Clinical Treatment } \\
\text { Required (n; \%) }\end{array}$ \\
\hline \multicolumn{3}{|l|}{ Routine blood test } \\
\hline Hemoglobin & $684 \llbracket 95.8 \% \rrbracket$ & $58 \varangle 8.1 \% \rrbracket$ \\
\hline Platelets & $98 \rrbracket 13.7 \% \rrbracket$ & $0 \otimes 0 \bigotimes$ \\
\hline \multicolumn{3}{|l|}{ Liver function test } \\
\hline Alanine aminotransferase & $85 \rrbracket 11.9 \% \rrbracket$ & $0 \otimes 0 \bigotimes$ \\
\hline $\begin{array}{l}\text { Aspartate } \\
\text { aminotransferase }\end{array}$ & 134ه18.8\%】 & $0 \otimes 0 \bigotimes$ \\
\hline Albumin & $688 \triangle 96.5 \% \square$ & $71 \otimes 9.9 \% \bigotimes$ \\
\hline \multicolumn{3}{|l|}{ Renal function test } \\
\hline Creatinine & $323 \rrbracket 45.2 \% \rrbracket$ & $0 \otimes 0 \bigotimes$ \\
\hline Blood urea nitrogen & 135ه18.9\%区 & $0 \otimes 0 \bigotimes$ \\
\hline \multicolumn{3}{|l|}{$\begin{array}{l}\text { Inflammation indicators } \\
\text { test }\end{array}$} \\
\hline $\begin{array}{l}\text { Erythrocyte sedimentation } \\
\text { rate }\end{array}$ & $230 \rrbracket 32.2 \% \rrbracket$ & $0 \otimes 0 \otimes$ \\
\hline C-reactive protein & $129 \rrbracket 2.7 \% \rrbracket$ & $0 \otimes 0 \bigotimes$ \\
\hline \multicolumn{3}{|l|}{ Electrolytes } \\
\hline Serum sodium & $73 \otimes 10.2 \% \rrbracket$ & $0 \otimes 0 \bigotimes$ \\
\hline $\begin{array}{l}\text { Serum potassium serum } \\
\text { potassium }\end{array}$ & $200 \rrbracket 28.0 \% \rrbracket$ & $24 \rrbracket 3.4 \% \rrbracket$ \\
\hline Serum calcium & 295『41.3\%区 & Oه०囚 \\
\hline
\end{tabular}

TKA: total knee Arthroplasty

Table 3. Postoperative Blood Transfusion for Patients with Abnormal Hemoglobin after TKA Surgery 


\begin{tabular}{|llll|}
\hline Factor & $\begin{array}{l}\text { Treatment } \\
\text { Group }(\mathbf{n}=\mathbf{5 8})\end{array}$ & $\begin{array}{l}\text { No Treatment } \\
\text { Group }(\mathbf{n = 6 2 6})\end{array}$ & P value \\
\hline Age (years) & $74.45 \pm 4.86$ & $66.55 \pm 10.86$ & 0.001 \\
\hline Sex $(\mathrm{n})$ & & & 0.067 \\
\hline Male & 20 & 148 & \\
\hline Female & 38 & 478 & \\
\hline BMl (kg/m $\left.{ }^{2}\right)$ & $22.37 \pm 3.40$ & $24.40 \pm 4.02$ & 0.495 \\
\hline Smoking: $\mathrm{n}(\%)$ & $19(32.8 \%)$ & $15(2.4)$ & 0.137 \\
\hline Alcohol use: $\mathrm{n}(\%)$ & $28(48.3)$ & $252(40.3)$ & 0.235 \\
\hline Diabetes mellitus: $\mathrm{n}(\%)$ & $5(8.6 \%)$ & $71(11.3 \%)$ & 0.504 \\
\hline High blood pressure: $\mathrm{n}(\%)$ & $23(39.75)$ & $234 \llbracket 37.4 \rrbracket$ & 0.732 \\
\hline Preoperative hb level $(\mathrm{g} / \mathrm{L})$ & $103.91 \pm 4.34$ & $125.05 \pm 11.05$ & $<0.001$ \\
\hline Estimated blood loss $(\mathrm{mL})$ & $426.88 \pm 306.54$ & $194.49 \pm 147.62$ & $<0.001$ \\
\hline Operative time (minutes) & $170.90 \pm 49.43$ & $137.10 \pm 41.32$ & 0.013 \\
\hline
\end{tabular}

BMI:body mass index, TKA: total knee Arthroplasty, HB: Hemoglobin

Table 4. Risk Factors for Postoperative Blood Transfusion in Patients Undergoing TKA Surgery

\begin{tabular}{|llll|}
\hline Risk factor & odds ratio & $95 \%$ confidence interval & P value \\
\hline Age & 1.148 & $1.068-1.234$ & $<0.001$ \\
\hline $\begin{array}{l}\text { Preoperative } \\
\text { hb level }\end{array}$ & 0.665 & $0.590-0.748$ & $<0.001$ \\
\hline $\begin{array}{l}\text { Estimated } \\
\text { blood loss }\end{array}$ & 1.008 & $1.005-1.011$ & $<0.001$ \\
\hline Operative time & 1.007 & $0.996-1.019$ & 0.210 \\
\hline
\end{tabular}

$\mathrm{Hb}$ : Hemoglobin

Table 5. Clinical Characteristics of Patients Who Required Postoperative Albumin Supplement 


\begin{tabular}{|llll|}
\hline Factor & $\begin{array}{l}\text { Treatment } \\
\text { Group }(\mathbf{n}=71)\end{array}$ & $\begin{array}{l}\text { No Treatment } \\
\text { Group (n=617) }\end{array}$ & P value \\
\hline Age (years) & $69.61 \pm 12.02$ & $67.08 \pm 10.48$ & 0.212 \\
\hline Sex (n) & & & $<0.001$ \\
\hline Male & 31 & 149 & \\
\hline Female & 40 & 468 & \\
\hline BMI (kg/m $\left.{ }^{2}\right)$ & $22.98 \pm 3.29$ & $24.35 \pm 4.05$ & 0.331 \\
\hline Smoking: $\mathrm{n}(\%)$ & $28(39.4 \%)$ & $147(23.8 \%)$ & 0.004 \\
\hline Alcohol use: $\mathrm{n}(\%)$ & $38(53.5 \%)$ & $246(39.9 \%)$ & 0.027 \\
\hline Diabetes mellitus: $\mathrm{n}(\%)$ & $6(8.5 \%)$ & $71(11.5 \%)$ & 0.439 \\
\hline High blood pressure: $\mathrm{n}(\%)$ & $22(31.0 \%)$ & $238(38.6 \%)$ & 0.212 \\
\hline Preoperative albumin $(\mathrm{g} / \mathrm{L})$ & $36.74 \pm 2.47$ & $42.50 \pm 3.80$ & $<0.001$ \\
\hline Estimated blood loss (mL) & $354.51 \pm 274.90$ & $196.66 \pm 155.29$ & $<0.001$ \\
\hline Operative time (minutes) & $212.11 \pm 23.36$ & $131.12 \pm 36.26$ & $<0.001$ \\
\hline
\end{tabular}

Table 6. Risk Factors for Patients Requiring Postoperative Albumin Supplementation

\begin{tabular}{|llll|}
\hline Risk factor & Odds ratio & $95 \%$ confidence interval & P value \\
\hline Sex & 2.566 & $0.587-11.206$ & 0.210 \\
\hline Smoking & 0.711 & $0.162-3.128$ & 0.652 \\
\hline Alcohol use & 1.359 & $0.607-3.045$ & 0.456 \\
\hline Estimated blood loss & 1.004 & $1.003-1.006$ & 0.037 \\
\hline Operative time & 1.011 & $1.004-1.018$ & $<0.001$ \\
\hline preoperative Albumin & 0.700 & $0.620-0.791$ & $<0.001$ \\
\hline
\end{tabular}

Table 7. Clinical Characteristics of Patients Who Required Postoperative Potassium Supplement 


\begin{tabular}{|llll|}
\hline Factor & $\begin{array}{l}\text { Treatment } \\
\text { Group }(\mathbf{n = 2 4 )}\end{array}$ & $\begin{array}{l}\text { No Treatment } \\
\text { Group }(\mathbf{n = 1 7 6})\end{array}$ & P value \\
\hline Age (years) & $69.29 \pm 6.87$ & $66.88 \pm 11.64$ & 0.218 \\
\hline Sex (n) & & & 0.144 \\
\hline Male & 2 & 42 & \\
\hline Female & 22 & 134 & \\
\hline BMI (kg/m $\left.{ }^{2}\right)$ & $20.82 \pm 1.84$ & $24.24 \pm 4.39$ & 0.006 \\
\hline Smoking: $\mathrm{n}(\%)$ & $4(\%)$ & 380 & 0.578 \\
\hline Alcohol use: $\mathrm{n}(\%)$ & $10(5)$ & 750 & 0.930 \\
\hline Diabetes mellitus: $\mathrm{n}(\%)$ & $2(\%)$ & $13(\%)$ & 0.869 \\
\hline High blood pressure: $\mathrm{n}(\%)$ & $15(\%)$ & $74(\%)$ & 0.059 \\
\hline Preoperative Potassium(g/L) & $3.66 \pm 0.23$ & $4.27 \pm 0.42$ & 0.02 \\
\hline Estimated blood loss $(\mathrm{mL})$ & $287.50 \pm 218.82$ & $215.45 \pm 185.80$ & 0.189 \\
\hline Operative time (minutes) & $144.58 \pm 53.79$ & $139.29 \pm 42.42$ & 0.098 \\
\hline
\end{tabular}

Table 8. Risk Factors for Patients Requiring Postoperative Potassium Supplementation

\begin{tabular}{|llll|}
\hline Risk factor & Odds ratio & $\mathbf{9 5} \%$ confidence interval & P value \\
\hline BMI & 0.615 & $0.479-0.789$ & $<0.001$ \\
\hline Preoperative Potassium & 0.001 & $0.000-0.009$ & $<0.001$ \\
\hline
\end{tabular}

Table 9. Cutoff Values of Risk Factors for Patients Requiring Postoperative Clinical Treatment 


\begin{tabular}{|lllllll|}
\hline Treatment & Risk factors & $\begin{array}{l}\text { Cut-off } \\
\text { value }\end{array}$ & Sensitivity & Specificity & Auc & $\begin{array}{l}\text { P } \\
\text { value }\end{array}$ \\
\hline Transfusion & Age & 69.5 & $87.9 \%$ & $61.3 \%$ & 0.767 & $<0.001$ \\
& Estimated blood loss & 225 & $77.6 \%$ & $75.7 \%$ & 0.839 & $<0.001$ \\
\hline \multirow{2}{*}{ Albumin } & Preoperative hb level & 111.5 & $91.9 \%$ & $86.2 \%$ & 0.933 & $<0.001$ \\
\hline & Estimated blood loss & 225 & $77.6 \%$ & $75.7 \%$ & 0.839 & $<0.001$ \\
\hline \multirow{2}{*}{ potassium } & Operative time & 152.5 & $63.8 \%$ & $69.6 \%$ & 0.696 & $<0.001$ \\
\hline & preoperative Albumin & 42.85 & $43.8 \%$ & $74.1 \%$ & 0.592 & 0.02 \\
& BMl & 21.89 & $73.9 \%$ & $83.3 \%$ & 0.793 & 0.04 \\
\hline & $\begin{array}{l}\text { Preoperative } \\
\text { potassium }\end{array}$ & 3.68 & $93.8 \%$ & $75 \%$ & 0.912 & 0.028 \\
\hline
\end{tabular}

BMI body mass index, AUC area under the curve 